\title{
Increased airway epithelial and T-cell apoptosis in COPD remains despite smoking cessation
}

\author{
S. Hodge, G. Hodge, M. Holmes and P.N. Reynolds
}

ABSTRACT: There is heterogeneity in the propensity of smokers to develop chronic obstructive pulmonary disease (COPD), and improved treatment strategies are hindered by limited understanding of COPD pathogenesis, especially as distinct from the effects of smoking per se. Although apoptosis is essential for tissue homeostasis, increased apoptosis may cause tissue damage and inflammation.

This study addressed whether airway T-lymphocytes and airway epithelial cells (AEC) show an increased likelihood of undergoing apoptosis in COPD and if this was related to smoking.

Apoptosis (7-amino-actinomycin D, Annexin, single-stranded DNA and caspase), Bcl-2, Bax and p53 were assessed in cells obtained from bronchial bushing and bronchoalveolar lavage from exand continuing smokers with COPD, and nonsmoking controls, using flow cytometry.

A mean $87 \%$ increase in apoptosis of AEC and a $103 \%$ increase in T-lymphocyte apoptosis were found in COPD. There were no significant differences in apoptosis of AEC between current and ex-smokers with COPD.

Apoptosis may contribute to chronic obstructive pulmonary disease pathogenesis, and continued excess apoptosis after smoking cessation may offer a new target for therapeutic interventions. Whether the persistence of increased apoptosis after smoking cessation results from changes in the pulmonary milleau after years of noxious insult, or whether some individuals have a natural predisposition toward increased apoptosis and possible development of chronic obstructive pulmonary disease remains to be determined.

KEYWORDS: Apoptosis, bronchial brushing, bronchoalveolar lavage, chronic obstructive pulmonary disease, flow cytometry, smoking

hronic obstructive pulmonary disease (COPD) arises as a result of noxious injury to the lungs, most commonly due to cigarette smoking. The overall impact of pharmacological therapies on the long-term outlook for patients with COPD is modest at best, and new therapeutic options are needed, based on improved knowledge of the underlying pathogenic mechanisms.

Key to the development of improved therapies is a greater understanding of the pathogenesis of COPD, especially as distinct from the effects of smoking per se. Therapies that address the ongoing abnormalities remaining after smoking cessation would be of great benefit. In this regard, disorders of apoptosis (programmed cell death) are increasingly recognised as having an important role in disease pathogenesis. Disorders in the apoptotic process associated with tissue injury have been seen in several conditions including emphysema [1-3], pulmonary fibrosis $[4,5]$, cystic fibrosis [6] and obliterative bronchiolitis [7]. Limited studies have shown increased lymphocyte and endothelial apoptosis in COPD $[1-3,8]$, raising the possibility that unregulated apoptosis may play a role in the pathogenesis of this disease, but apoptosis of airway epithelial cells has not been systematically examined.

Although apoptosis is important for eliminating excess activated inflammatory cells, excess apoptosis of these cells and/or airway epithelial cells may be counterproductive, resulting in diminished host defences. The current authors have previously found that peripheral blood T-cells from COPD patients have an increased propensity to undergo apoptosis compared with cells from healthy subjects [9]. The authors thus sought to determine whether these peripheral abnormalities may be reflected in T-cells derived from the lungs where the pathological significance

\section{AFFILIATIONS}

Dept of Thoracic Medicine, Royal Adelaide Hospital and Hanson Institute, Adelaide,

South Australia, Australia.

\section{CORRESPONDENCE}

S. Hodge

Lung Research Laboratory

Dept of Thoracic Medicine

Royal Adelaide Hospital

North Terrace

Adelaide

South Australia 5001

Australia

Fax: 61881616043

E-mail: sandy.hodge@imvs.

sa.gov.au

Received:

June 282004

Accepted after revision:

November 052004

SUPPORT STATEMENT

S. Hodge is supported by an Australian Lung Foundation/ Boehringer-Ingelheim Chronic Airflow Limitation Fellowship. P.N. Reynolds is supported by an NHMRC Clinical Career Development Award. 
may be even greater. In addition, uncleared apoptotic cells may undergo secondary necrosis with discharge of injurious cell contents, tissue damage and chronic inflammation [10]. The current authors have previously shown that pulmonary macrophages from COPD subjects have a defect in their ability to phagocytose apoptotic airway epithelial cells in ex vivo assays [11], which may thus predispose subjects with COPD to an accumulation of apoptotic material in the airways [12].

To the current authors' knowledge there have been no studies of apoptosis in discrete cell types from heterogeneous cell populations obtained from bronchoalveolar lavage (BAL) and bronchial brushing in COPD, especially with regard to the possible effects of smoking status. In the current study, apoptosis in well-characterised COPD subjects, including exsmokers and continuing smokers, and nonsmoking normal controls was assessed.

\section{MATERIALS AND METHODS \\ Immunological reagents}

The following monoclonal antibodies (MAbs) and reagents were employed: CD45(PE-CY5), CD3(PE-CY5) (Immunotech/ Coulter, Marseille, France), CD3(PE), p53(PE) (BD Biosciences, CA, USA), Bcl-2(FITC) (Dako, Glostrup, Denmark), Bax (unconjugated) (Oncogene Science, NY, USA), rabbit antimouse immunoglobulin (Ig)G (RAM, PE; BD Biosciences), IgG1(PE) (as negative control; BD Biosciences), Annexin $\mathrm{V}$ (FITC) (BD Biosciences), 7-amino-actinomycin D (7-AAD) (Sigma, Sydney, Australia), CaspACE FITC-VAD-FMK (Promega, WI, USA), unconjugated antibody to singlestranded DNA (ssDNA) (MAB3299 (F7-26); Chemicon, CA, USA) and goat anti-mouse IgM(FITC) (Rockland, PA, USA).

\section{Subject population}

COPD patients undergoing fibreoptic bronchoscopy for diagnostic purposes (predominantly because of lesions suspicious of carcinoma on chest radiograph) were invited to participate in the study and fully informed consent was obtained. There was no exacerbation of COPD for 6 weeks prior to involvement in the study. Ethics approval was obtained from the Royal Adelaide Hospital, Adelaide, Australia. The diagnosis of moderate COPD was established using the Global Initiative for Chronic Obstructive Lung Disease criteria [13] of a relevant history and post-bronchodilator forced expiratory volume in one second (FEV1) 30-80\% of predicted and FEV1/forced vital capacity $<70 \%$.

Bronchial brushings were collected from 29 patients with COPD (brushing group) (table 1). Sixteen patients were ex-smokers ( $>1$ yr abstinence) and 13 current smokers.

BAL was collected from 20 patients with COPD (BAL group) (there was overlap between this group and the brushing group due to unsuitability or unavailability of occasional specimens of BAL or bronchial brushing) (table 1). In total, 13 patients were ex-smokers ( $>1$ yr abstinence) and seven current smokers.

Specimens were also obtained from 20 nonsmoking volunteers (table 1). In total, 10 of these controls were healthy, recruited volunteers with no history of airways disease and 10 were undergoing bronchoscopy for other clinically indicated reasons. Typically, these were patients with chronic cough, which proved to be related to gastro-oesophageal reflux or sinus disease, or with minor probable haemoptysis for which no pulmonary cause could be found. These subjects enabled the power of the study to be improved and fulfilled the criteria for normal control (normal lung function, no evidence of any lung disease, no history of asthma or allergy, nonsmokers, normal bronchoscopy). Most of the controls were included in both the BAL and brushing studies, apart from a few subjects in whom either the BAL or brush was inadequate for technical reasons. Importantly, there were no significant differences between these two groups of controls for any of the parameters investigated in this study. The first phase of the study investigated apoptosis in brushing-derived epithelial cells

TABLE 1 Demographic characteristics of the population studied

\begin{tabular}{|c|c|c|c|c|}
\hline Subjects & Control & COPD & COPD ex-smokers & COPD current smokers \\
\hline \multicolumn{5}{|l|}{ Brushing group } \\
\hline Subjects $\mathrm{n}$ & 20 & 29 & 16 & 13 \\
\hline Age yrs & $60 \pm 13$ & $67 \pm 9$ & $69 \pm 9$ & $65 \pm 10$ \\
\hline Smoking pack-yr & 0 & $60 \pm 26$ & $69 \pm 30$ & $47 \pm 21$ \\
\hline FEV $1 \%$ pred & $104.2 \pm 10.4$ & $65.4 \pm 18.3$ & $57.3 \pm 19.6$ & $50.5 \pm 16.2$ \\
\hline \multicolumn{5}{|l|}{ FVC BAL group } \\
\hline Subjects $\mathrm{n}$ & 20 & 20 & 13 & 7 \\
\hline Age yr & $61 \pm 12$ & $69 \pm 8$ & $70 \pm 8$ & $68 \pm 8$ \\
\hline Smoking pack-yr & 0 & $66 \pm 32$ & $73 \pm 31$ & $33 \pm 17$ \\
\hline FEV $1 \%$ pred & $102.1 \pm 11.6$ & $53.1 \pm 17.3$ & $50.3 \pm 19.1$ & $46.0 \pm 8.4$ \\
\hline FVC \% pred & $102.6 \pm 15.4$ & $79.4 \pm 13.9$ & $77.9 \pm 14.1$ & $73.5 \pm 6.7$ \\
\hline
\end{tabular}

Data presented as mean \pm SD. COPD: chronic obstructive pulmonary disease; FEV1: forced expiratory volume in one second; \% pred: \% predicted; FVC: forced vital capacity; BAL: bronchoalveolar lavage. 
and BAL-derived T-cells. For this phase of the study the two control groups were pooled into a single control group.

\section{Bronchoscopy procedure}

Fibreoptic bronchoscopy was performed as previously described [11]. Brushings and BAL were obtained from the right-sided airways, unless there was other pathology in this area, when the left-sided airways were sampled. Particular attention was given to use a minimal amount of lignocaine $(100 \mathrm{mg})$ in the airways in view of the adverse effect it has on cell viability [14].

\section{Preparation of ex vivo samples}

Cells were prepared as previously described [11, 15] and resuspended to a cell count of $4 \times 10^{5}$ cells $\cdot \mathrm{mL}^{-1}$ with RPMI 1640 media. Investigation of apoptosis of airway T-cells and epithelial cells was performed within $1 \mathrm{~h}$. For investigation of Bcl-2, Bax and p53 by BAL-derived T-cells, BAL samples were stimulated with phytohaemagglutin for $18 \mathrm{~h}$, as previously reported [9].

\section{Measurement of LDH levels in BAL}

When the cells of a tissue become damaged or necrotic, their contents leak out into the extracellular fluid. Thus, the existence of secondary necrosis in the airways can be inferred by the presence of lactic dehydrogenase (LDH) in BAL. LDH levels were measured in BAL from the 10 volunteer control subjects and 10 of the COPD subjects (five smokers, five exsmokers) using a Beckman Synchron $C^{\mathrm{R}}$ System (Beckman Instruments Brea, CA, USA), following instructions supplied with the reagent kit.

\section{Identification of cell types}

Surface staining to identify cell types was carried out using flow-cytometry, as previously described $[16,17]$ and is shown in figures 1 and 2. Leukocytes were excluded from analysis of airway epithelial cells based on bright staining with CD45 (fig. $1 b)$. T-cells were gated based on known staining characteristics with CD3 PC5 versus side scatter (fig. 2b). As both 7-AAD and PC5 fluoresce in fluorescence channel 3 (FL3), CD3 PE (FL2) versus side scatter was used for identification of T-cells prior to staining with 7-AAD.

\section{Apoptosis of BAL-derived leukocytes and brushing-derived airway epithelial cells}

Annexin $\mathrm{V}$ staining

Early in apoptosis, phosphatidylserine (PTS) is translocated to the outer cell membrane and can be identified by binding of Annexin V, a ligand for PTS. Staining with Annexin V was performed as previously described [16, 18].

7-AAD staining

Staining with 7-AAD was used to quantify apoptosis as previously described [18]. Absolute numbers of 7-AAD positive cells were calculated then adjusted for differences in lymphocyte or airway epithelial cell numbers for COPD and control groups.

Staining of apoptotic cells with MAbs to ssDNA

To investigate the possibility that removal of airway epithelial cells from the basement membrane caused membrane damage
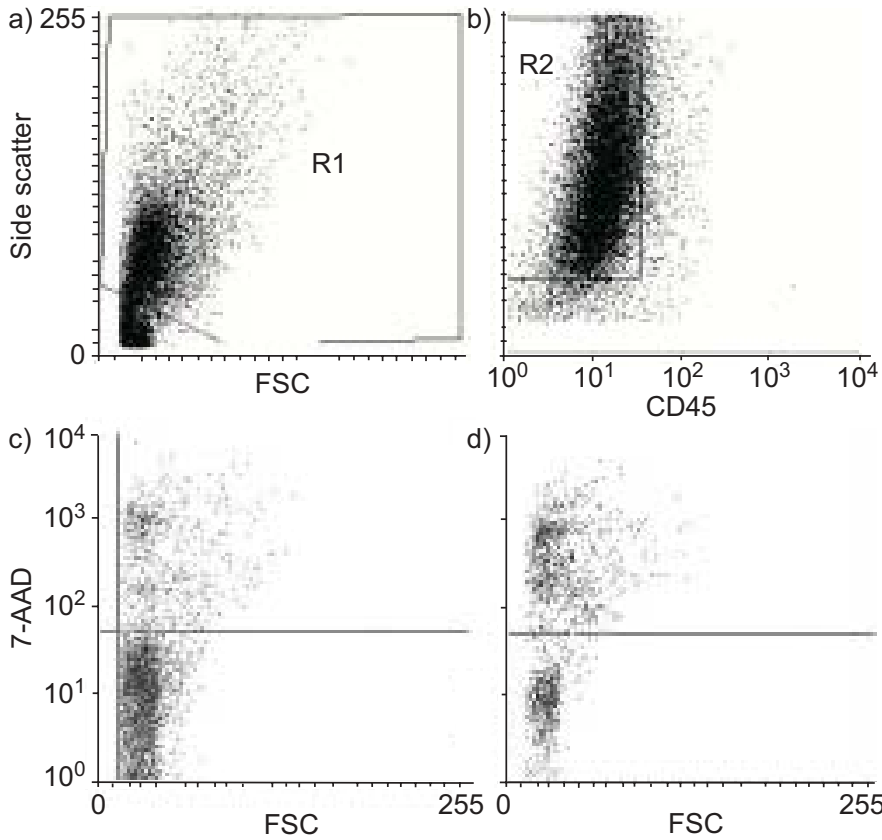

FIGURE 1. Flow cytometry gating strategies for brushing-derived airway epithelial cells. a) Red blood cells and debris were excluded from Region (R)1 based on forward scatter (FSC) and side scatter characteristics. All subsequent analysis was carried out on cells from this region. b) Contaminating leukocytes identified by bright staining with CD45 and excluded from airway epithelial cells in R2. All subsequent analysis carried out on cells from R1 and R2. c and d) 7-AAD staining of apoptotic brushing-derived airway epithelial cells. Representative dot plots showing FSC versus 7-AAD staining to distinguish viable (7-AAD negative staining) and apoptotic (7-AAD bright staining) epithelial cells. Control subject: $14 \%$ apoptotic (c); chronic obstructive pulmonary disease subject: $32 \%$ apoptotic (d).

during the bronchoscopy procedure, it was important to establish alternative methods for assessing apoptosis that did not rely on changes to the cell membrane. Antibody MAB3299 is specifically reactive with ssDNA (i.e. does not recognise DNA in double stranded conformations). Staining was performed following manufacturer's instructions.

\section{Staining of apoptotic cells for active caspases}

Sequential activation of caspases plays a critical role in the induction of apoptosis. CaspACE FITC-VAD-FMK is an FITCconjugate of the cell permeable caspase inhibitor VAD-FMK. This structure allows delivery of the inhibitor into the cell where it binds to caspase, serving as a marker for apoptosis. Cells were stained with $100 \mu \mathrm{L}, 10 \mu \mathrm{M}$ CaspACE FITC-VADFMK, followed by staining with surface markers for cell type identification.

\section{Effects of glucocorticosteroids on apoptosis of airway-derived T-cells and epithelial cells}

Glucocorticosteroids (GCS) have been reported to induce apoptosis of airway epithelial cells and T-cells [19, 20]. To investigate this possibility in a preliminary fashion, COPD subjects with similar lung function were grouped based on GCS or bronchodilator treatment, (brushing group: 13 GCS-treated and 16 subjects using bronchodilator medication only; BAL 

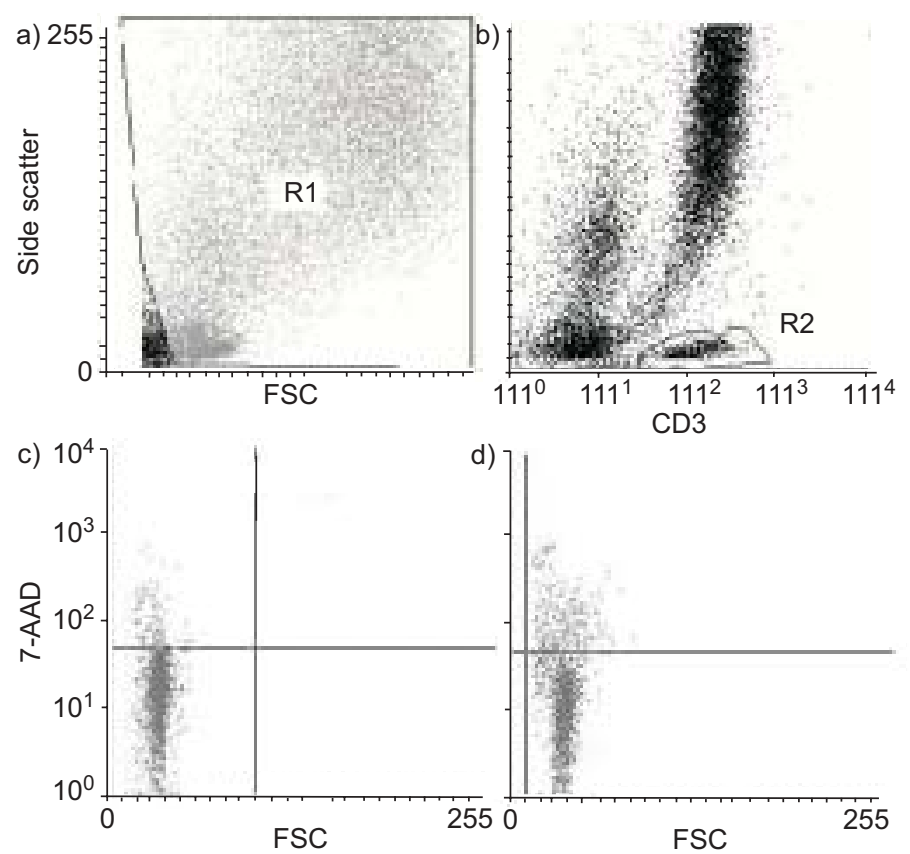

FIGURE 2. Flow cytometry gating strategies for bronchiolar lavage-derived Tcells. a) Red blood cells and debris were excluded from Region (R) 1 based on forward scatter (FSC) and side scatter characteristics. All subsequent analysis carried out on cells from this region. b) T-cells gated in R2 based on bright staining with $\mathrm{CD} 3$ and low side scatter. All subsequent analysis carried out on cells from R1 and R2. (CD45 PE was also applied to confirm the gating (data not shown)). $\mathrm{c}$ and d) 7-AAD staining of apoptotic brushing-derived airway epithelial cells. Representative dot plots showing FSC versus 7-AAD staining to distinguish viable (7-AAD negative staining) and apoptotic (7-AAD bright staining) epithelial cells. Control subject: $5 \%$ apoptotic (c); chronic obstructive pulmonary disease subject: $18 \%$ apoptotic (d).

group: 11 GCS-treated and nine subjects using bronchodilator only). Apoptosis was assessed as described above, and results compared for the two groups. However, the subjects were heterogeneous with respect to the type of inhaled steroid being used, the dose, and furthermore no guarantees could be given as regard their compliance with medication. Thus, a more definitive analysis of the effects of GCS on apoptosis in COPD awaits further study.

\section{Effect of age on apoptosis of airway-derived T-cells and epithelial cells}

There have been some reports of increased apoptosis of peripheral blood T-cells with increasing age [21]. To investigate this possibility the control group was divided into two age categories, those aged $<50$ yrs and those aged 55-70 yrs. Apoptosis was calculated as described above, and results compared for the two groups.

\section{Effect of smoking on apoptosis of airway-derived T-cells and epithelial cells}

COPD subjects with similar lung function were grouped based on smoking status (fig. 1), apoptosis of airway epithelial cells and T-cells assessed as described above, and results compared for the two groups.

\section{Bax, Bcl-2 and $p 53$ expression by airway-derived T-cells and epithelial cells in COPD}

Staining for cell type identification was carried out as described above. Bax, Bcl-2 and p53 were stained using a direct flow-cytometric staining technique. Aliquots of $1 \times 10^{6}$ cells were washed with calcium and magnesium-free PBS and $200 \mu \mathrm{L}$ of membrane permeabilising solution $(0.5 \%$ Triton $\mathrm{X}$ 100 (Sigma), $0.2 \mu \mathrm{g} \cdot \mathrm{mL}^{-1} \mathrm{Na}_{2}$ EDTA, 2H2O (APS Chemicals, NSW, Australia), 1\% BSA in PBS) added to the cell pellet for 15 min. Cells were stained with MAbs to Bax $(5 \mu \mathrm{L}), \operatorname{Bcl}-2(10$ $\mu \mathrm{L})$ or p53 $(5 \mu \mathrm{L})$. Irrelevant antibodies of the same isotypes were used as negative controls. For Bcl-2 and p53, analysis of unwashed, unfixed cells was carried out immediately by flow-cytometry. For Bax, cells were further stained with $5 \mu \mathrm{L}$ PE-conjugated rat-anti-mouse Mab, washed and analysed by flow-cytometry (fig. 1).

\section{Statistical analysis}

The Mann-Whitney U-test was used to analyse data. p-Values $<0.05$ were considered significant.

\section{RESULTS}

\section{Apoptosis of BAL-derived leukocytes and brushing-derived airway epithelial cells}

As previously reported for peripheral blood T-cells and neutrophils [9, 18], there was good correlation between Annexin V staining and 7-AAD staining for the detection of apoptosis of brushing-derived epithelial cells $\left(R^{2}=0.8\right)$.

Typical scatter patterns of forward scatter versus 7-AAD are presented in figure 1 and typical histograms presented in figure 3. The percentage of apoptotic airway epithelial cells was significantly higher for patients with COPD than controls for each of the techniques used (table 2). The mean increase in apoptosis across the techniques was $87 \%$ (calculated as (COPD-control)/control). In agreement with results from analysis of percentage changes, there were significantly more absolute numbers of apoptotic airway epithelial cells in COPD (control: $0.06 \times 10^{9} \cdot \mathrm{L}^{-1}$ versus COPD: $0.10 \times 10^{9} \cdot \mathrm{L}^{-1} ; \mathrm{p}=0.03$ ).

Disruption of epithelial cells from the basement membrane has been reported to induce apoptosis [22]. In addition, the local anaesthetic, lignocaine, has been reported to diminish epithelial cell viability, in vitro [14]. Staining with Annexin V and 7AAD relies on changes to the cell membrane, but it was considered that any confounding factor due to technical issues would similarly affect the COPD and control groups. Nevertheless, to investigate whether cell removal or use of lignocaine (used at a minimum dose, $100 \mathrm{mg}$ ) caused artefactual membrane damage during the bronchoscopy procedure, techniques were further applied to specifically detect apoptosis, based on changes that did not involve the cell membrane (ssDNA and caspase) (fig. 3). These again confirmed higher rates of apoptosis in COPD.

Consistent with findings for peripheral blood-derived T-cells from COPD patients [9], BAL-derived T-cells showed increased apoptosis, with a mean increase of $103 \%$ across the techniques used (table 2). The absolute numbers of apoptotic T-cells (adjusted for total lymphocte numbers) were significantly increased in COPD (control: $0.003 \times 10^{9} \cdot \mathrm{L}^{-1}$ versus COPD: $0.02 \times 10^{9} \cdot \mathrm{L}^{-1} ; \mathrm{p}=0.01$ ). 


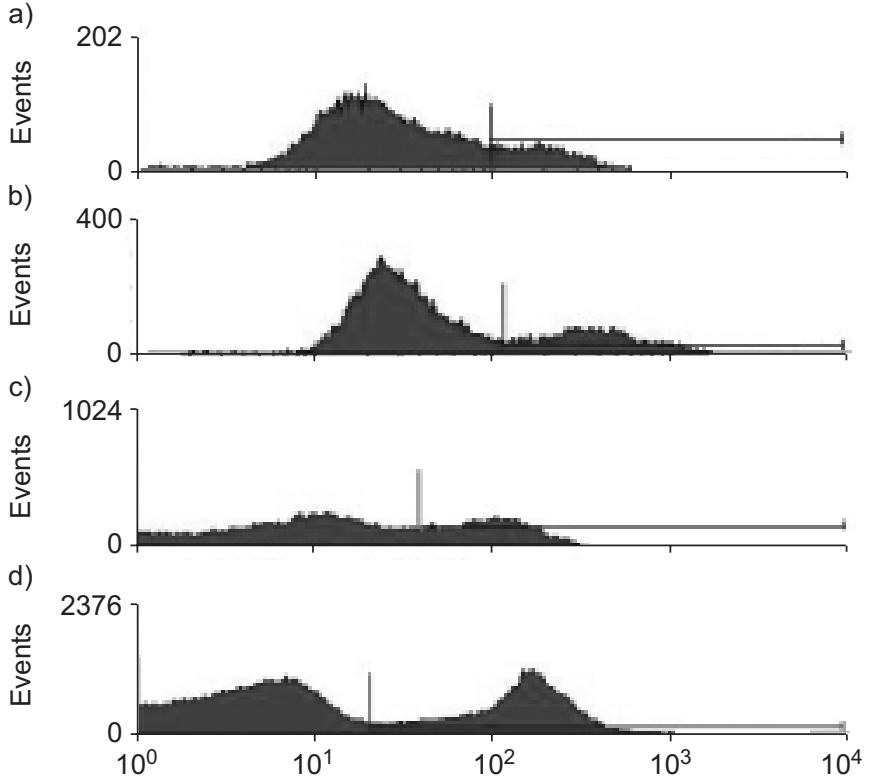

FIGURE 3. Apoptosis of brushing-derived airway epithelial cells measured by different techniques. Representative histograms show: a) Annexin $V$ staining of phosphatidylserine on the cell membrane (20.0\% apoptotic); b) 7-AAD staining due to nonfunctional membrane pump action (22.3\% apoptotic); c) staining of intranuclear single-standed DNA in early apoptosis (27.3\% apoptotic); and d) staining of intracellular caspase activation in early apoptosis (37.6\% apoptotic).

TABLE 2 Apoptosis of airway-derived T-cells and epithelial cells in chronic obstructive pulmonary disease (COPD)

\begin{tabular}{lcll} 
Method & Control & COPD & p-value \\
\hline Epithelial cell & & & \\
7-AAD & $18.2 \pm 12.1$ & $33.2 \pm 14.1$ & $0.008^{\#}$ \\
Annexin & $15.0 \pm 8.6$ & $31.0 \pm 10.0$ & $0.008^{\#}$ \\
ssDNA & $14.0 \pm 7.2$ & $36.3 \pm 15.8$ & $0.001^{\#}$ \\
Caspase & $37.6 \pm 15.6$ & $65.1 \pm 6.2$ & $0.004^{\#}$ \\
T-cell & & & \\
7-AAD & $9.1 \pm 6.0$ & $21.3 \pm 9.7$ & $0.001^{\#}$ \\
Annexin & $11.7 \pm 9.9$ & $27.9 \pm 12.4$ & $0.002^{\#}$ \\
ssDNA & $21.1 \pm 13.0$ & $51.0 \pm 7.9$ & $0.0005^{\#}$ \\
Caspase & $17.8 \pm 9.9$ & $28.2 \pm 13.5$ & $0.042^{\#}$ \\
\hline
\end{tabular}

Data (\% of cells exhibiting positive staining measured by flow cytometry) presented as mean \pm SD. SSDNA: single-stranded DNA. \#: significant difference from control group.

\section{Effects of age on apoptosis of airway-derived T-cells and epithelial cells}

There were no significant changes in apoptosis of airway epithelial cells or BAL-derived T-cells with increasing age (epithelial cells: controls aged <50 yrs, 7-AAD 20.0 $\pm 11.6 \%$ (mean $\pm \mathrm{SD}$ ) versus controls aged 55-75 yrs, $20.0 \pm 14.6 \%$; Tcells: controls aged $<50$ yrs, 7 -AAD $19.9 \pm 11.6 \%$ versus controls aged $55-75$ yrs $17.2 \pm 12.6 \%)$. Annexin V staining supported these findings (data not shown).
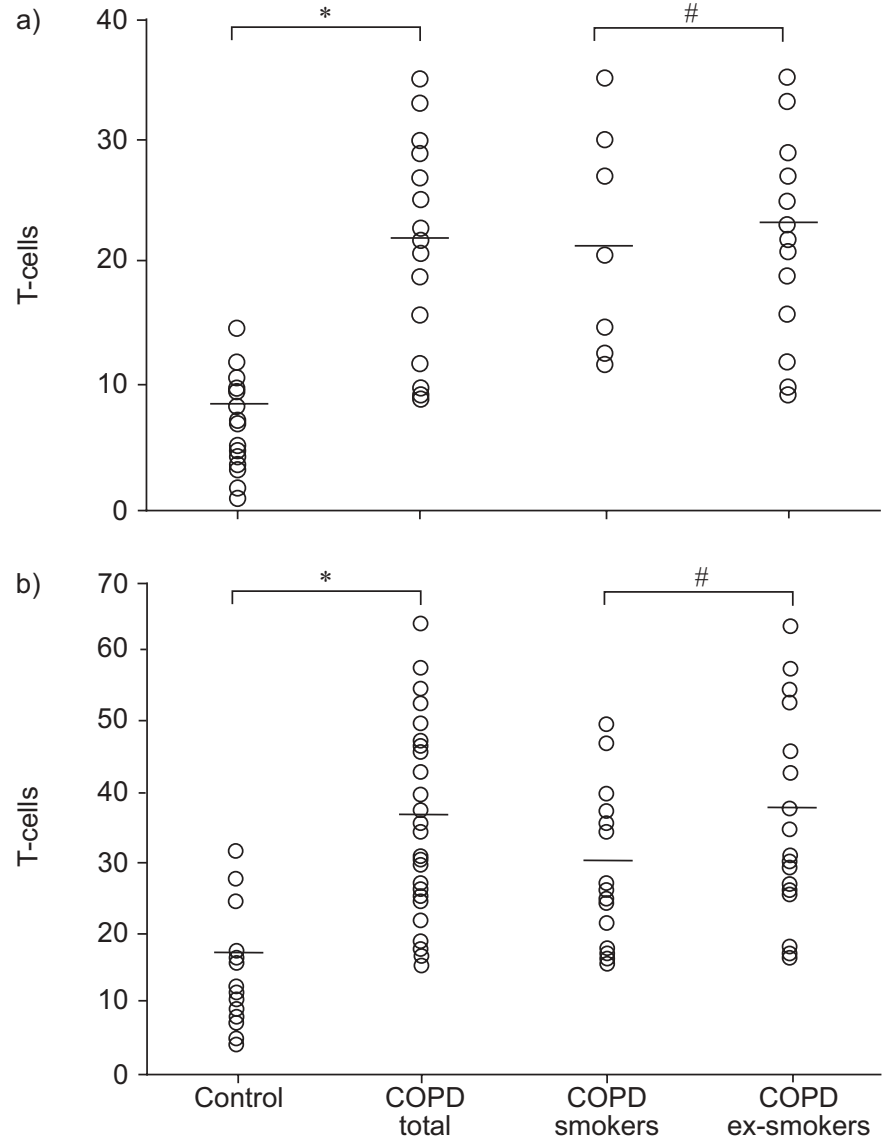

FIGURE 4. Apoptosis of a) bronchoalveolar lavage-derived T-cells and b) brushing-derived airway epithelial cells measured by 7-AAD staining and flow cytometry in controls, chronic obstructive pulmonary disease (COPD) subjects (total group), and smokers and ex-smokers with COPD. *: significant difference between COPD and control groups $p \leqslant 0.05$; \#: no significant difference between smokers and ex-smokers with COPD.

\section{Effects of smoking on apoptosis of airway-derived T-cells and epithelial cells}

There were no significant differences in apoptosis of airway epithelial cells or BAL-derived T-cells between smoking and nonsmoking COPD subjects (fig. 4).

\section{Bax, Bcl-2 and p53 expression by airway-derived T-cells and epithelial cells in COPD}

Expression of Bax, Bcl-2 and p53 was analysed by flowcytometry. There was no significant change in the percentage of airway epithelial cells or T-cells expressing Bcl-2 or Bax in COPD compared with controls (table 3). Similarly, the median fluorescence intensity of staining was not significantly different (data not shown). P53 expression by BAL-derived CD3+ T-cells and brushing-derived airway epithelial cells was significantly increased in COPD compared with controls (table 3).

\section{LDH levels in BAL in COPD}

To determine if secondary necrosis of airway-derived cells was apparent, LDH levels were measured in BAL from the 10 normal volunteer subjects and 10 COPD subjects (five of the smokers and five of the ex-smokers). LDH levels were 


\begin{tabular}{llcc}
\hline TABLE 3 & $\begin{array}{l}\text { Expression of apoptosis-related proteins by } \\
\text { airway-derived T-cells and epithelial cells in } \\
\text { chronic obstructive pulmonary disease (COPD) }\end{array}$ \\
Cell type & Control group & CoPD & p-value \\
\hline Epithelial cell & & & \\
P53 & $18.4 \pm 7.1$ & $28.1 \pm 8.5$ & $0.005^{\star}$ \\
Bcl-2 & $22.3 \pm 7.5$ & $23.2 \pm 8.7$ & NS \\
Bax & $46.3 \pm 9.4$ & $52.4 \pm 9.6$ & NS \\
T-cell & & & \\
P53 & $23.9 \pm 8.8$ & $33.0 \pm 9.2$ & $0.045^{\star}$ \\
Bcl-2 & $26.6 \pm 13.2$ & $22.9 \pm 7.9$ & NS \\
Bax & $41.1 \pm 20.7$ & $40.2 \pm 7.3$ & NS \\
\hline
\end{tabular}

Data (\% of cells exhibiting positive staining measured by flow cytometry) presented as mean values \pm SD. NS: nonsignificant. *: significant difference from control group.

significantly higher in BAL from COPD subjects compared with controls $\left(1.9 \pm 2.9 \mathrm{IU} \cdot \mathrm{L}^{-1}\right.$ versus $\left.0.1 \pm 0.3 \mathrm{IU} \cdot \mathrm{L}^{-1} ; \mathrm{p}=0.040\right)$.

\section{DISCUSSION}

Apoptosis of BAL-derived lymphocytes and brushing-derived airway epithelial cells was significantly increased in COPD. Apoptosis of airway epithelial cells was not affected by increasing age or by smoking status among those with COPD. By using a number of independent assays for apoptosis the current authors are confident that all stages of apoptosis were detected and that results were not confounded by the inclusion of necrotic cells. To the current authors' knowledge, apoptosis has not previously been quantified in discrete leukocyte subsets from heterogenous cell populations obtained from the lungs of patients with COPD. However, a few studies have reported increased apoptosis of alveolar or bronchial epithelial cells and lymphocytes in bronchial biopsies from patients with COPD [3, 8, 23]. Importantly, these studies showed that apoptosis might at least partially account for the loss of alveolar wall structures and formation of bullae in emphysema.

Lung damage in COPD involves proximal and distal airways, as well as alveoli. BAL is a useful technique, which samples the inflammatory milieu of the most distal airways, and alveoli, thus providing ideal samples of cells from a broader area than is the case with small biopsies. In addition to BAL, bronchial brushings were obtained from airways of 2-4 mm diameter. In both cases the high sensitivity of the flow-cytometry technique used in the current study allows for the acquisition of meaningful data from several of the subsets of cells present, even in the presence of a high predominance of any one particular cell type (e.g. macrophages in BAL). Although the entire airway tree is involved, these airways contribute substantially to the airflow obstruction that is the physiological hallmark of COPD.

Cigarette smoking has been shown to induce apoptosis of airway epithelial cells [20]. However, in the present study, cessation of smoking did not prevent the increased rates of apoptosis in COPD. This may be a reflection of the ongoing abnormalities remaining after smoking cessation in COPD, rather than the effects of cigarette smoking per se. Several studies have shown that inflammation of the airway mucosa of COPD patients persist even after smoking cessation [24-27]. In addition, alveolar destruction, emphysematous lung enlargement, permeability coefficient and pulmonary arterial pressure are unchanged by cessation of cigarette smoking [28, 29]. Taken together with the current authors' findings, these data suggest that the trend toward normalisation of the natural decline in lung function seen with smoking cessation [30, 31] may not accurately reflect the persistent disordered apoptosis and inflammation in the airways in COPD.

The increased apoptosis that persists despite smoking cessation suggests that factors other than cigarette smoke must play a role once COPD disease is established. In this regard, factors that relate to perpetuation of the chronic inflammatory response in COPD may also contribute to increased apoptotis in the airways. These include increased oxidative stress and release of proteolytic enzymes as a result of increased numbers of neutrophils, cytotoxic T-cells, activation of Fas, tumour necrosis factor- $\alpha$ and transforming growth factor- $\beta$ pathways, and inflammatory responses to colonisation of the airways with bacteria [4, 12, 20,32]. GCS have been reported to induce apoptosis of airway epithelial cells in vitro [19, 20]. However, within the constraints of the present study no additional epithelial cell apoptosis was observed for the group of COPD subjects who were treated with inhaled GCS, although there was a trend toward higher apoptosis of BAL-derived T cells in the GCS group. Further study of greater numbers of patients on defined steroid preparations and dosages is needed to fully investigate the association between GCS and apoptosis.

Under normal conditions, apoptosis is followed by rapid phagocytosis of apoptotic cells. The current authors have previously reported that alveolar macrophages from COPD subjects are deficient in their ability to phagocytose apoptotic airway epithelial cells [11]. An increased rate of apoptosis of epithelial cells in the airways has now been shown in the current study. This may outweigh the phagocytic capacity of alveolar macrophages and result in defective clearance and accumulation of apoptotic cells, leading to secondary necrosis with release of toxic cell contents, tissue damage and chronic inflammation [12], leading to progression of the pathological processes in COPD. Increased levels of $\mathrm{LDH}$ in BAL verified the presence of secondary necrosis in the airways in COPD. This increase was significant and possibly even an underestimate of the total amount of secondary necrosis, as some necrotic cells may have been phagocytosed prior to complete release of $\mathrm{LDH}$ to the lumen.

Apoptosis is accompanied by the activation of various death pathways, including the mitochondrial-mediated pathway. This pathway is regulated by the Bcl family of pro-apoptotic proteins (e.g. p53, Bax), as well as some proteins (e.g. $\mathrm{Bcl} 2)$ that can inhibit apoptosis [33-35]. To further investigate the causes of increased apoptosis in COPD, the current authors investigated the expression of $\mathrm{Bcl}, \mathrm{Bax}, \mathrm{p} 53$ and caspases in airwayderived T-cells and epithelial cells. Previous studies have shown a good correlation between Western blot (the gold standard) and flow-cytometry for the detection of these apoptosis-related proteins [36]. Flow-cytometry was used because of its unique ability to identify the specific cell types 
expressing these proteins. In the peripheral blood the current authors have previously observed a significant decrease in expression of Bcl-2 for T-cells from GCS-treated patients with COPD (unpublished data). The lack of any significant difference in expression of Bcl-2 or Bax by airway-derived Tcells in the present study was, therefore, surprising. However, the findings of increased expression of p53 and of caspases by airway derived T-cells and epithelial cells have important implications in COPD. P53-regulated apoptosis can be induced by oxidative stress $[37,38]$. Increased oxidative stress has been reported in COPD [39] and may thus contribute to increased p53, and subsequently increased apoptosis. Consistent with these findings in patients with COPD, increased p53 expression has been associated with apoptosis in type II pneumocytes in diffuse alveolar damage [40], in bronchial and alveolar epithelial cells in bleomycin-induced pulmonary fibrosis in mice, and interstitial pneumonia and idiopathic pulmonary fibrosis in humans [41-43]. Further studies to determine the levels of apoptosis-inducing cytokines in BAL will help to further characterise the mechanisms involved.

In summary, increased apoptosis of airway-derived epithelial cells associated with up-regulation of caspase activity and p53 expression suggests that apoptosis may play a significant role in the chronic inflammation and defective repair processes that are the hallmarks of chronic obstructive pulmonary disease pathogenesis. Increased apoptosis of T-cells may lead to a diminished immune response to infective organisms and contribute to the increased frequencies of infection that are associated with chronic obstructive pulmonary disease. Importantly, the increase in apoptosis appears to persist even after smoking cessation. Whether this continued abnormality results from a phenotypic change in the pulmonary milieu after years of noxious insult, or whether there is a subgroup of the population with a natural predisposition toward increased apoptosis and therefore the development of chronic obstructive pulmonary disease, remains to be determined. Either way, the presence of this ongoing abnormality may provide a basis for new therapeutic approaches aimed at reducing apoptosis.

\section{ACKNOWLEDGEMENTS}

The authors acknowledge the invaluable contribution of the Bronchoscopy Unit of the Thoracic Medicine Dept, Royal Adelaide Hospital.

\section{REFERENCES}

1 Kasahara Y, Tuder RM, Cool CD, Lynch DA, Flores SC, Voelkel NF. Endothelial cell death and decreased expression of vascular endothelial growth factor and vascular endothelial growth factor receptor 2 in emphysema. Am J Respir Crit Care Med 2001; 163: 737-744.

2 Kasahara Y, Tuder RM, Taraseviciene-Stewart L, et al. Inhibition of VEGF receptors causes lung cell apoptosis and emphysema. J Clin Invest 2000; 106: 1311-1319.

3 Majo J, Ghezzo H, Cosio MG. Lymphocyte population and apoptosis in the lungs of smokers and their relation to emphysema. Eur Respir J 2001; 17: 946-953.

4 Hagimoto N, Kuwano K, Miyazaki H, et al. Induction of apoptosis and pulmonary fibrosis in mice in response to ligation of Fas antigen. Am J Respir Cell Mol Biol 1997; 17: 272-278.
5 Hagimoto N, Kuwano K, Nomoto Y, Kunitake R, Hara N. Apoptosis and expression of Fas/Fas ligand mRNA in bleomycin-induced pulmonary fibrosis in mice. Am J Respir Cell Mol Biol 1997; 16: 91-101.

6 Vandivier RW, Fadok VA, Ogden CA, et al. Impaired clearance of apoptotic cells from cystic fibrosis airways. Chest 2002; 121: Suppl. 3, 89S.

7 Hansen PR, Holm AM, Svendsen UG, Olsen PS, Andersen CB. Apoptosis and formation of peroxynitrite in the lungs of patients with obliterative bronchiolitis. J Heart Lung Transplant 2000; 19: 160-166.

8 Yokohori N, Aoshiba K, Nagai A. Increased levels of cell death and proliferation in alveolar walls in patients with pulmonary emphysema. Chest 2004; 125: 626-632.

9 Hodge SJ, Hodge GL, Reynolds PN, Scicchitano R, Holmes M. Increased production of TGF-beta and apoptosis of $\mathrm{T}$ lymphocytes isolated from peripheral blood in COPD. Am J Physiol Lung Cell Mol Physiol 2003; 285: L492-L499.

10 Kuwano K, Hagimoto N, Kawasaki M, et al. Essential roles of the Fas-Fas ligand pathway in the development of pulmonary fibrosis. J Clin Invest 1999; 104: 13-19.

11 Hodge S, Hodge G, Scicchitano R, Reynolds PN, Holmes M. Alveolar macrophages from subjects with chronic obstructive pulmonary disease are deficient in their ability to phagocytose apoptotic airway epithelial cells. Immunol Cell Biol 2003; 81: 289-296.

12 Hart SP, Haslett C, Dransfield I. Recognition of apoptotic cells by phagocytes. Experimentia 1996; 52: 950-956.

13 Pauwels RA, Buist AS, Ma P, Jenkins CR, Hurd SS, and the GOLD Scientific Committee, Global Initiative for Chronic Obstructive Lung Disease. National Institutes of Health, National Heart, Lung and Blood Institute. 2001; 2701: 7.

14 Kelsen SG, Mardini IA, Zhou S, Benovic JL, Higgins NC. A technique to harvest viable tracheobronchial epithelial cells from living human donors. Am J Respir Cell Mol Biol 1992; 7: 66-72.

15 Hodge SJ, Hodge GL, Holmes M, Reynolds PN. Flow cytometric characterization of cell populations in bronchoalveolar lavage and bronchial brushings from patients with chronic obstructive pulmonary disease. Cytometry 2004; 61B: 27-34.

16 Hodge G, Hodge S, Han P. Increased levels of apoptosis of leukocyte subsets in cultured PBMCs compared to whole blood as shown by Annexin $\mathrm{V}$ binding: relevance to cytokine production. Cytokine 2000; 12: 1763-1768.

17 Han P, Hodge G, Story C, Xu X. Phenotypic analysis of functional T-lymphocyte subtypes and natural killer cells in human cord blood: relevance to umbilical cord blood transplantation. Br J Haematol 1995; 89: 733-740.

18 Hodge GL, Flower R, Han P. Optimal storage conditions for preserving granulocyte viability as monitored by Annexin $\mathrm{V}$ binding in whole blood. J Immunol Methods 1999; 225: 27-38.

19 Dorscheid DR, Wojcik KR, Sun S, Marroquin B, White SR. Apoptosis of airway epithelial cells induced by corticosteroids. Am J Respir Crit Care Med 2001; 164: 1939-1947.

20 Vernooy JH, Dentener MA, van Suylen RJ, Buurman WA, Wouters EF. Intratracheal instillation of lipopolysaccharide in mice induces apoptosis in bronchial epithelial cells: no 
role for tumour necrosis factor-alpha and infiltrating neutrophils. Am J Respir Cell Mol Biol 2001; 24: 569-576.

21 Pahlavani MA, Vargas DA. Aging but not dietary restriction alters the activation-induced apoptosis in rat $\mathrm{T}$ cells. FEBS Lett 2001; 491: 114-118.

22 Frisch SM, Francis H. Disruption of epithelial cell-matrix interactions induces apoptosis. J Cell Biol 1994; 124: 619-626.

23 Segura-Valdez L, Pardo A, Gaxiola M, Uhal BD, Becerril C, Selman M. Upregulation of gelatinases A and B, collagenases 1 and 2, and increased parenchymal cell death in COPD. Chest 2000; 117: 684-694.

24 Turato G, Di Stefano A, Maestrelli P, et al. Effect of smoking cessation on airway inflammation in chronic bronchitis. Am J Respir Crit Care Med 1995; 152: 1262-1267.

25 Rutgers SR, Postma DS, ten Hacken NH, et al. Ongoing airway inflammation in patients with COPD who do not currently smoke. Thorax 2000; 55: 12-18.

26 Domagala-Kulawik J, Maskey-Warzechowska M, Kraszewska I, Chazan R. The cellular composition and macrophage phenotype in induced sputum in smokers and ex-smokers with COPD. Chest 2003; 123: 1054-1059.

27 Pride NB. Smoking cessation: effects on symptoms, spirometry and future trends in COPD. Thorax 2001; 56: Suppl. 2, 7-10.

28 Wright JL, Hobson JE, Wiggs B, Pare PD, Hogg JC. Airway inflammation and peribronchiolar attachments in the lungs of nonsmokers, current and ex-smokers. Lung 1988; 166: 277-286.

29 Wright JL, Sun JP. Effect of smoking cessation on pulmonary and cardiovascular function and structure: analysis of guinea pig model. J Appl Physiol 1994; 76: 2163-2168.

30 Fletcher C, Peto R. The natural history of chronic airflow obstruction. BMJ 1977; 1: 1645-1648.

31 Burchfiel CM, Marcus EB, Curb JD, et al. Effects of smoking and smoking cessation on longitudinal decline in pulmonary function. Am J Respir Crit Care Med 1995; 151: 1778-1785.
32 D’Agostini F, Balansky RM, Izzotti A, Lubet RA, Kelloff GJ, De Flora S. Modulation of apoptosis by cigarette smoke and cancer chemoproventive agents in the respiratory tract of rats. Carcinogenesis 2001; 22: 375-380.

33 Kastan MB, Onyekwere O, Sidransky D, Vogelstein B, Craig RW. Participation of p53 protein in the cellular response to DNA damage. Cancer Res 1991; 51: 6304-6311.

34 Lowe SW, Schmitt EM, Smith SW, Osborne BA, Jacks T. p53 is required for radiation-induced apoptosis in mouse thymocytes. Nature 1993; 362: 847-849.

35 Cory S. Regulation of lymphocyte survival by the bcl-2 gene family. Annu Rev Immunol 1995; 13: 513-543.

36 van Stijn A, Kok A, van der Pol MA, et al. A flow cytometric method to detect apoptosis-related protein expression in minimal residual disease in acute myeloid leukemia. Leukemia 2003; 17: 780-786.

37 Yin Y, Terauchi Y, Solomon GG, et al. Involvement of p85 in p53-dependent apoptotic response to oxidative stress. Nature 1998; 391: 707-710.

38 Ueda S, Masutani H, Nakamura H, Tanaka T, Ueno M, Yodoi J. Redox control of cell death. Antioxid Redox Signal 2002; 4: 405-414.

39 Rahman I, Morrison D, Donaldson K, MacNee W. Systemic oxidative stress in asthma, COPD, and smokers. Am J Respir Crit Care Med 1996; 154: 1055-1060.

40 Guinee DJ, Fleming M, Hayashi T, et al. Association of p53 and WAF1 expression with apoptosis in diffuse alveolar damage. Am J Pathol 1996; 149: 531-538.

41 Kuwano K, Hagimoto N, Tanaka T, et al. Expression of apoptosis-regulatory genes in epithelial cells in pulmonary fibrosis in mice. J Pathol 2000; 190: 221-229.

42 Kuwano K, Kunitake R, Kawasaki M, et al. P21Waf1/Cip1/ Sdi1 and p53 expression in association with DNA strand breaks in idiopathic pulmonary fibrosis. Am J Respir Crit Care Med 1996; 154: 477-483.

43 Kunitake R, Kuwano K, Miyazaki H, et al. Expression of p53, p21 (Waf1/Cip1/Sdi1) and Fas antigen in collagen vascular and granulomatous lung diseases. Eur Respir J 1998; 12: 920-925. 